Es bestehen bei summarischer Prüfung auch keine ernstlichen Zweifel an der Verhältnismäßigkeit der Regelung. Es liegt auf der Hand, dass die Vorlage eines Originalattests für den Nachweis eines Befreiungsgrundes i.S.v. \$2 Abs. 2 S. 1 Nr. 3 der 3. SARS-CoV-2-EindV eine höhere Verlässlichkeit bietet als die Vorlage einer Kopie, auf der sich eine Fälschung gegebenenfalls schwerer erkennen lässt. Soweit der ASt. darauf verweist, es gebe Situationen, in denen der Befreiungsgrund offensichtlich oder sonst eine niedrigschwelligere Kontrolldichte vertretbar sei, wie beispielsweise bei einem ,,von Zeugen beobachteten oder gegebenenfalls filmisch dokumentierten akuten Asthmaanfall", vermag dies die Befugnis des Verordnungsgebers, eine sämtliche Fälle erfassende zudem eine ärztliche Beurteilung zugrunde legende pauschalierende Regelung zu treffen, nicht in Frage zu stellen.

Aber auch wenn man davon ausgeht, dass sich die Rechtmäßigkeit der Regelung mit Blick auf die Kürze der vorliegend zur Verfügung stehenden Zeit nicht hinreichend beurteilen lässt, der Ausgang des Rechtsbehelfs in der Hauptsache mithin offen ist, geht jedenfalls die danach vorzunehmende Folgenabwägung zulasten des ASt. aus.

Die Versagung des von dem ASt. begehrten vorläufigen Rechtsschutzes nach $₫ 47$ Abs. $6 \mathrm{VwGO}$ hat für diesen zur Folge, dass er den ihm ärztlich attestierten Befreiungsgrund von der Pflicht zum Tragen einer Mund-Nasen-Bedeckung durch Vorlage des ärztlichen Zeugnisses bis zu einer Entscheidung in einem Hauptsacheverfahren im Original nachzuweisen hat. Der damit einhergehende Eingriff in seine allgemeine Handlungsfreiheit i.S.v. Art. 2 Abs. 1 GG ist von geringem Gewicht. Das Mitführen des Original-Attests führt zu keiner nennenswerten Belastung. Der Gefahr des Verlustes oder einer Beschädigung des Originals kann durch eine entsprechend sorgfältige Behandlung seitens des ASt. entgegengewirkt werden. Gegebenenfalls mag er dieses Original auch in einer Klarsichthülle aufbewahren und zur Kontrolle vorzeigen und erforderlichenfalls aushändigen. Sollte tatsächlich ein Verlust oder eine zur Unbrauchbarkeit führende Beschädigung eintreten, dürfte es auch ohne unverhältnismäßigen Aufwand möglich sein, die Ausstellung eines Ersatz-Attests zu erreichen.

Würde die vom ASt. (noch zulässigerweise) angegriffene Vorschrift hingegen vorläufig außer Vollzug gesetzt, so hätte dies zur Folge, dass nicht nur im Fall des ASt., sondern allgemein die Vorlage einer Kopie des ärztlichen Zeugnisses bis auf weiteres ausreichen würde. Dies würde die Kontrolle der Echtheit des jeweiligen ärztlichen Zeugnisses erschweren und die Gefahr eines Missbrauchs erhöhen. Der gegenwärtige Stand der Pandemie lässt es indes nicht zu, auf das grundsätzlich wirksame Mittel der Verwendung einer Mund-Nasen-Bedeckung auch in Fällen zu verzichten, in denen dies nicht auch tatsächlich gerechtfertigt ist. [...]

https://doi.org/10.1007/s00350-021-6067-8

\section{Offenbarung der Diagnose zur Befreiung vom Tragen einer sog. Mund-Nase-Bedeckung; sensible Gesundheitsdaten}

CoronaV3EindV BB §2 Abs. 2 S. 2; IfSG §28, 28a, 32; VwGO $\S 47$ Abs. 6

Der $\$ 2$ Abs. 2 S. 2 CoronaV3EindV BB ist vorläufig außer Vollzug gesetzt, als dass ärztliche Zeugnis danach die konkret $\mathrm{zu}$ benennende gesundheitliche

Eingesandt von Rechtsanwalt Prof. Dr. iur. Martin Stellpflug, Berlin; bearbeitet von Rechtsanwalt Bernd Klemp, LL.M.,

Fachanwalt für Medizinrecht und Fachanwalt für Versicherungsrecht, Bietmann Rechtsanwälte Steuerberater PartmbB,

Martinstraße 22-24, 50667 Köln, Deutschland
Beeinträchtigung (Diagnose) sowie konkrete Angaben beinhalten muss, warum sich hieraus eine Befreiung von der Tragepflicht ergibt.

OVG Berlin-Brandenburg, Beschl. v. 4.1.2021-OVG 11 S 132/20

Problemstellung: Die Regelung des $₫ 2$ Abs. 2 S. 2 CoronaV3EindV BB beinhaltet, dass das schriftliche ärztliche Zeugnis darüber, dass die Verwendung einer Mund-Nasen-Bedeckung aus gesundheitlichen Gründen nicht möglich oder unzumutbar sei - neben Daten zur Identifizierung - auch , ,die konkret zu benennende gesundheitliche Beeinträchtigung (Diagnose) sowie konkrete Angaben beinhalten muss, warum sich hieraus eine Befreiung von der Tragepflicht ergibt.

Damit müssen für die Person sensible Daten einem unbestimmt großen Personenkreis, der über keine entsprechenden medizinischen Fachkenntnisse verfügt, offenbart werden. Dabei sind bezüglich der Weitergabe dieser Daten durch die ,,vor Ort" überprüfende Stelle, mithin in der Regel eine nicht-öffentlichen Stelle, keine Grenzen gesetzt. Weder regelt die CoronaV3EIndV BB ein verpflichtendes Stillschweigen noch ein entsprechendes Bußgeld. Demgegenüber sei nach der Entscheidung des Gerichts der Nachweispflicht für die Befreiung durch ein ärztliches Attest, das Namen und Geburtsdatum enthält und somit eine Identifikation erlaubt, Genüge getan.

Die Situation ist vergleichbar mit der Situation der Nachweispflicht bei der Arbeitsunfähigkeitsbescheinigung gegenüber dem Arbeitgeber, wo die Diagnose in der Bescheinigung nicht enthalten ist, da es sich hierbei um eine private Angelegenheit handelt.

Bernd Klemp

Zum Sachverhalt: Der ASt., der nach unbestritten gebliebenen eigenen Angaben ausweislich eines ärztlichen Attestes v. 14.8.2020 aus gesundheitlichen Gründen vom Tragen einer sog. Mund-NaseBedeckung befreit ist, wendet sich im Wege einstweiliger Anordnung gemäß $₫ 47$ Abs. $6 \mathrm{VwGO}$ dagegen, dass das ärztliche Zeugnis, mit dem die Befreiung von der Tragepflicht einer Mund-NasenBedeckung ,,vor Ort" nachzuweisen ist ( $\$ 2$ Abs. 2 S. 1 Nr. 3 der 3 SARS-CoV-2-EindV), nach $₫ 2$ Abs. 2 S. 2 der 3. SARS-CoV-2EindV die konkret zu benennende gesundheitliche Beeinträchtigung (Diagnose) sowie konkrete Angaben beinhalten muss, warum sich hieraus eine Befreiung von der Tragepflicht ergibt.

$\$ 2$ der 3. SARS-CoV-2-EindV lautet:

[...]

(2) Von der Pflicht zum Tragen einer Mund-Nasen-Bedeckung sind folgende Personen befreit:

[...]

3. Personen, denen die Verwendung einer Mund-Nasen-Bedeckung wegen einer Behinderung oder aus gesundheitlichen Gründen nicht möglich oder unzumutbar ist; dies ist vor Ort durch ein schriftliches ärztliches Zeugnis im Original nachzuweisen.

Das ärztliche Zeugnis nach S. 1 Nr. 3 muss mindestens den vollständigen Namen und das Geburtsdatum, die konkret zu benennende gesundheitliche Beeinträchtigung (Diagnose) sowie konkrete Angaben beinhalten, warum sich hieraus eine Befreiung von der Tragepflicht ergibt. Sofern im Einzelfall eine Dokumentation der Befreiung von der Tragepflicht erforderlich ist, darf die Tatsache, dass das ärztliche Zeugnis vorgelegt wurde, die ausstellende Ärztin oder der ausstellende Arzt sowie ein eventueller Gültigkeitszeitraum des ärztlichen Zeugnisses in die zu führenden Unterlagen aufgenommen werden; die Anfertigung einer Kopie des ärztlichen Zeugnisses ist nicht zulässig.

Zur Begründung seines Antrags macht der ASt. im Wesentlichen geltend:

Die erst mit der 3. SARS-CoV-2-EindV eingeführte Regelung in $\$ 2$ Abs. 2 S. 2 der 3. SARS-CoV-2-EindV [...] verletze sein Grundrecht auf informationelle Selbstbestimmung. Diese Daten, die der ärztlichen Schweigepflicht unterlägen, seien als Gesundheitsdaten sensible Daten i.S.d. \$3 Abs. 9 BDSG und genössen insoweit besonderen Schutz. Eine Befreiung von der Maskenpflicht aus gesundheit- 
lichen Gründen komme auch in psychischer Hinsicht in Fällen des sexuellen Missbrauchs bei Fesselung bzw. Knebelung des Opfers und hierbei erlebten Ohnmachtsgefühlen in Betracht. Es sei weder erforderlich noch angemessen, dass er (auch) diese Daten zum Nachweis seiner Befreiung quasi täglich jedermann ,,vor Ort“, z.B. in Einzelhandelsgeschäften, Apotheken, im öffentlichen Nahverkehr, aber auch gegenüber seinem Arbeitgeber offenbaren müsse. Die Personen „,vor Ort" seien mangels medizinischer Fachkenntnisse ohnehin zu keiner inhaltlichen Überprüfung des ärztlichen Attestes befähigt, so dass die Vorschrift im Verhältnis zur bisherigen Regelung keinen zusätzlichen Nutzen bringe. Er indes habe ein starkes subjektives Interesse daran, dass seine Diagnose und ihre konkreten Folgen (privaten) Dritten nicht zur Kenntnis gelangt. Konkret befürchte er, dass seine hochsensiblen Gesundheitsdaten, wenn er das Attest mit der ,Klarfassung“ seiner Krankheit im Supermarkt, in der Apotheke, in der Poststelle etc. vorzeigen müsse, durch Mund-Propaganda im Dorf schnell ,die Runde machten“.

$[\ldots]$

Aus den Gründen: Der Antrag auf Erlass einer einstweiligen Anordnung gemäß $₫ 47$ Abs. 6 VwGO hat Erfolg.

1. Der Antrag ist zulässig.

Gemäß $₫ 47$ Abs. 1 Nr. 2 VwGO i.V. mit $₫ 4$ Abs. 1 Bbg VwGG entscheidet das OVG im Rahmen seiner Gerichtsbarkeit auf Antrag über die Gültigkeit von anderen (nicht von Nr. 1 erfassten) im Rang unter dem Landesgesetz stehenden Rechtsvorschriften und damit auch über die angegriffene Vorschrift in $\$ 2$ Abs. 2 S. 2 der 3. SARS-CoV-2EindV.

Der ASt. ist gemäß $\ 47$ Abs. $2 \mathrm{~S} .1 \mathrm{VwGO}$ antragsbefugt, da die in $\$ 2$ Abs. 2 S. 2 der 3. SARS-CoV-2-EindV geregelte Offenbarungspflicht für die Diagnose sowie für konkrete Angaben, warum sich hieraus eine Befreiung von der Tragepflicht ergibt, ihn jedenfalls in seinem durch Art. 2 Abs. 1 i. V. mit Art. 1 Abs. 1 GG geschützten Recht auf informationelle Selbstbestimmung verletzen kann.

2. Der Antrag ist auch begründet.

Nach $₫ 47$ Abs. 6 VwGO kann das Gericht auf Antrag eine einstweilige Anordnung erlassen, wenn dies zur Abwehr schwerer Nachteile oder aus anderen wichtigen Gründen dringend geboten ist. Prüfungsmaßstab im Verfahren nach $\$ 47$ Abs. 6 VwGO sind in erster Linie die Erfolgsaussichten des in der Hauptsache anhängigen Normenkontrollantrags, soweit sich diese im Verfahren des einstweiligen Rechtsschutzes bereits absehen lassen. Dabei erlangen die Erfolgsaussichten des Normenkontrollantrags eine umso größere Bedeutung für die Entscheidung im Eilverfahren, je kürzer die Geltungsdauer der in der Hauptsache angegriffenen Normen befristet und je geringer damit die Wahrscheinlichkeit ist, dass eine Entscheidung über den Normenkontrollantrag noch vor dem Außerkrafttreten der Normen ergehen kann.

Ergibt demnach die Prüfung der Erfolgsaussichten der Hauptsache, dass der Normenkontrollantrag voraussichtlich unzulässig oder unbegründet sein wird, ist der Erlass einer einstweiligen Anordnung nicht zur Abwehr schwerer Nachteile oder aus anderen wichtigen Gründen dringend geboten. Erweist sich dagegen, dass der Antrag zulässig und (voraussichtlich) begründet sein wird, so ist dies ein wesentliches Indiz dafür, dass der Vollzug bis zu einer Entscheidung in der Hauptsache suspendiert werden muss. In diesem Fall kann eine einstweilige Anordnung ergehen, wenn der (weitere) Vollzug vor einer Entscheidung im Hauptsacheverfahren Nachteile befürchten lässt, die unter Berücksichtigung der Belange des ASt., betroffener Dritter und/oder der Allgemeinheit so gewichtig sind, dass eine vorläufige Regelung mit Blick auf die Wirksamkeit und Umsetzbarkeit einer für den ASt. günstigen Hauptsachenentscheidung unaufschiebbar ist.

Lassen sich die Erfolgsaussichten des Normenkontrollverfahrens im Zeitpunkt der Entscheidung über den Eilantrag nicht (hinreichend) abschätzen, ist über den Erlass einer beantragten einstweiligen Anordnung im Wege einer Folgenabwägung zu entscheiden: Gegenüberzustellen sind die Folgen, die eintreten würden, wenn eine einstweilige Anordnung nicht erginge, das Hauptsacheverfahren aber Erfolg hätte, und die Nachteile, die entstünden, wenn die begehrte einstweilige Anordnung erlassen würde, das Normenkontrollverfahren aber erfolglos bliebe. Die für den Erlass der einstweiligen Anordnung sprechenden Erwägungen müssen die gegenläufigen Interessen dabei deutlich überwiegen, mithin so schwer wiegen, dass der Erlass der einstweiligen Anordnung - trotz offener Erfolgsaussichten der Hauptsache - dringend geboten ist (vgl. zum vorstehenden insgesamt: Senatsbeschluss vom 23.4.2020 - OVG 11 S 25/20 -, Rdnrn. 4-7, juris; OVG Schleswig-Holstein, Beschl. v. 9. 4.2020 - 3 MR 4/20 -, Rdnrn. 3-5, juris; BayVGH, Beschl. v. 30.3.2020 - 20 NE 20.632 -, juris Rdnrn. $31 \mathrm{ff}$., jeweils unter Hinweis auf BVerwG, Beschl. v. 25.2.2015 - 4 VR $5.14-$, juris, Rdnr. 12).

Hiernach ist der begehrte Erlass einer einstweiligen Anordnung dringend geboten. Der Senat vermag die Erfolgsaussichten des Rechtsbehelfs in der Hauptsache mit Blick auf die Kürze der Zeit nicht hinreichend zu beurteilen. Es lässt sich mit Blick auf die Eilbedürftigkeit der Sache nicht sicher feststellen, ob die angegriffene Offenbarungsbzw. Nachweispflicht zu Gesundheitsdaten einer Prüfung im Normenkontrollverfahren voraussichtlich standhalten wird. In Frage steht insofern bereits, ob der mit der angegriffenen Regelung verbundene datenschutzrechtliche Eingriff in $\iint 32,28$ Abs. 1, 28a Abs. 1 Nr. 2 IfSG i.d. F. v. 18.11.2020, der notwendige Schutzmaßnahmen in Form einer Maskenpflicht erlaubt, eine hinreichende Rechtsgrundlage findet. Die Erfolgsaussichten im Hauptsacheverfahren sind daher bei summarischer Prüfung als offen einzuschätzen. Die danach vorzunehmende Folgenabwägung geht zulasten des Ag. aus.

Die Versagung des von dem ASt. begehrten vorläufigen Rechtsschutzes nach $₫ 47$ Abs. $6 \mathrm{VwGO}$ hat für diesen zur Folge, dass er seine konkrete Diagnose und sich daraus ergebene Folgen an einer Vielzahl von Stellen - wie z.B. in Verkaufsstellen des Groß- und Einzelhandels (vgl. $\$ 8$ Abs. 4 Nr. 3), in öffentlichen Verkehrsmittels (vgl. \$15 Abs. 1), in Arbeits- und Betriebsstätten sowie Büro- und Verwaltungsgebäuden (vgl. \$20 Abs. 1), bei Versammlungen unter freiem Himmel ( $\$ 5$ Abs. 1 Nr. 3), bei religiösen Veranstaltungen (vgl. $\$ 16$ Abs. $1 \mathrm{Nr}$. 3), ,vor Ort", mithin in der Regel nicht-öffentlichen Stellen offenbaren muss, wobei es sich hierbei um personenbezogene Gesundheitsdaten handelt, die besonders sensibel sind und daher einem besonders hohen Datenschutz unterfallen. Dass auch Private zur Kontrolle der Atteste verpflichtet sind, ergibt sich bereits daraus, dass sie die Einhaltung der Verpflichtung zum Tragen einer Mund-Nasen-Bedeckung sicherzustellen haben und widrigenfalls eine Ordnungswidrigkeit begehen (vgl. z. B. \$24 Abs. 1 Nr. 9, 14, 21, 27 der 3. SARS-CoV-2-EindV).

Soweit der ASt. befürchtet, seine sensiblen Gesundheitsdaten könnten durch Mund-Propaganda im Dorf schnell „die Runde machen“, wenn er das Attest mit der „Klarfassung“" seiner Krankheit im Supermarkt, in der Apotheke, in der Poststelle etc. vorzeigen müsse, ist dies nicht von der Hand zu weisen. Denn die Verordnung selbst regelt nicht, dass die Personen, gegenüber denen der Nachweis „,vor Ort" zu erbringen ist, Stillschweigen über die erhobenen Gesundheitsdaten zu bewahren haben. Die Preisgabe der erhobenen Gesundheitsdaten ist nach der 3. SARSCoV-2-EindV - im Gegensatz zum Anfertigen einer Kopie des ärztlichen Zeugnisses (vgl. dort $\$ 24$ Abs. 1 Nr. 3) auch nicht bußgeldbewehrt. Anders als dies etwa für das Erfassen von Personendaten in einem Kontaktnachweis zum Zwecke der Kontaktnachverfolgung in $\$ 1$ Abs. 3 S. 4 der 3. SARS-CoV-2-EindV geschehen ist, finden sich für die erhobenen Gesundheitsdaten in der 3. SARS-CoV-2- 
EindV auch sonst keine Regelungen zur Zweckbindung der Datenverwendung. Eine damit von der Verordnung in Kauf genommene Preisgabe sensibler Gesundheitsdaten des vorgenannten Ausmaßes stellt einen gravierenden Eingriff in die Rechte des ASt. dar.

Der AG. hat nicht dargelegt, dass bei Erfolg des Antrags auf einstweiligen Rechtsschutz vergleichbare schwere Folgen in Rede stehen. Der Erlass der einstweiligen Anordnung hat nicht zur Folge, dass die Nachweispflicht für Personen, denen die Verwendung einer Mund-Nasen-Bedeckung wegen einer Behinderung oder aus gesundheitlichen Gründen nicht möglich oder unzumutbar ist, gänzlich entfällt. Vielmehr ist die Befreiung aus gesundheitlichen Gründen vor Ort - wie bis zum Erlass der 3. SARS-CoV-2-EindV - durch ein ärztliches Zeugnis, das Namen und Geburtsdatum enthält, d.h. eine Identifikation erlaubt, nachzuweisen. Soweit der AG. geltend macht, er habe die Anforderungen an das ärztliche Zeugnis präzisiert, um der Gefahr von Gefälligkeitsattesten ,wirksam vorzubeugen“, hat er diese Gefahr bereits nicht hinreichend belegt. Dabei ist zu berücksichtigen, dass die Maskenpflicht nur dann unterlaufen zu werden droht, wenn Gefälligkeitsatteste in nennenswertem Umfang im Raume stünden. Hierfür führt der AG. indes nichts an. Vielmehr verweist er zu Recht selbst darauf, das das Ausstellen unrichtiger Gesundheitszeugnisse nach $\$ 278$ StGB strafbewehrt ist. Soweit er auf verwaltungsgerichtliche Rechtsprechung (VG Würzburg, Beschl. v. 16.9.2020 - W 8 E 20.1301 -, juris, Rdnr. 22; VG Düsseldorf, Beschl. v. 25. 8. 2020 - 18 L 1608/20 -, juris, Rdnr. 37; BayVGH, Beschl. v. 26.10.2020 - 20 CE 20.2185 -, juris, Rdnr. 19) Bezug nimmt, betrifft diese bereits nicht die (aktuelle) Situation im Land Brandenburg. Unabhängig hiervon ist diese Rechtsprechung zu Anträgen auf Feststellung, dass eine Befreiung von der Maskenpflicht aus gesundheitlichen Gründen im Einzelfall besteht, ergangen. In diesen Fällen war die Prüfung jeweils nicht von privaten Stellen, sondern von einem ohnehin datenschutzrechtlichen Bindungen unterliegenden Gericht vorgenommen worden. Soweit darin im Einzelfall festgestellt wird, dass das Gericht ein ärztliches Attest als Nachweis für eine Befreiung aus gesundheitlichen Gründen nur dann für ausreichend überprüfbar hält, wenn dieses auch eine medizinische Diagnose und die sich hieraus ergebenden Folgen für das Tragen einer Mund-Nasen-Bedeckung beschreibt, bedeutet dies nicht zwangsläufig, dass eine wirksame Kontrolle des Befreiungstatbestandes die Vorlage eines solchen Attestes „,vor Ort“ überall dort, wo eine Maskenpflicht gilt, erfordert.

\section{Anmerkung zu OVG Berlin-Brandenburg, Beschl. v. 4.1.2021 - OVG 11 S 132/20:}

\section{Bernd Klemp}

Dass die Diagnose eine entscheidende Rolle für die Freistellung von der Pflicht zum Tragen einer Mund-NasenBedeckung spielt, steht außer Frage. Dass diese jedoch nicht in allen Situationen in dem Originalattest preisgegeben werden müsste, hat das Gericht richtigerweise erkannt.

Um Diskriminierungen zu vermeiden und das allgemeine Persönlichkeitsrecht zu schützen, ist es angemessen im öffentlichen Raum - u.a. bei alltäglichen Einkaufssituationen, in der Apotheke, in der Poststelle oder in öffentli-

Rechtsanwalt Bernd Klemp, LL.M.

Fachanwalt für Medizinrecht und Fachanwalt für Versicherungsrecht, Bietmann Rechtsanwälte Steuerberater PartmbB,

Martinstraße 22-24, 50667 Köln, Deutschland chen Verkehrsmitteln - auf die Offenbarung der Diagnose zu verzichten. Zum gegenwärtigen Zeitpunkt der Pandemie wird die Glaubhaftmachung der Freistellungssituation durch Nichtangabe der Diagnose im Originalattest nicht erschüttert, da es keine Anhaltspunkte gibt, dass Gefälligkeitsatteste von Ärzten ausgestellt würden.

Vergleichbar zu anderen Entscheidungen ${ }^{1}$, wo innerhalb des Schulbetriebs die Diagnose im Attest enthalten sein sollte, besteht dort nicht die Gefahr, dass datenschutzwidriger Umgang gegeben sei. Denn das Attest wird in einem geschützten Rahmen vorgezeigt, wo interne Vorschriften den Umgang von persönlichen Daten gewährleisten.

Daher wird es angemessen sein anhand Einzelfallbetrachtungen zu entscheiden, wann die Diagnose im Attest enthalten sein sollte und wann nicht. Im öffentlichen Raum, wo der datenschutzkonforme Umgang nicht gewährleistet werden kann, überwiegt der Schutz des allgemeinen Persönlichkeitsrechts des Freigestellten.

1) Z.B. OVG Münster, Beschl. v. 24.9.2020 - 13 B 1368/20.

\section{Voraussetzungen einer Sonderbedarfsfeststellung}

GG Art. 12 Abs. 1; SGB V §§95 Abs. 2, 101 Abs. 1 S. 1 Nr. 3, 285 Abs. 1, Abs. 3 S. 1; BedarfsplanungsRL §§36, 37; DSGVO Art. 6 Abs. 1 und 2

1. In einem Rechtsstreit über eine Sonderbedarfsanstellung ist die Beiladung des anzustellenden Arztes nicht erforderlich.

2. Bei der Entscheidung über eine Sonderbedarfszulassung oder -anstellung sind die Grundsätze über Vornahmeklagen anzuwenden. Danach sind grundsätzlich alle Tatsachenänderungen bis zur mündlichen Verhandlung der letzten Tatsacheninstanz und alle Rechtsänderungen bis zum Abschluss der Revisionsinstanz zu berücksichtigen.

3. Auch eine Zusatzweiterbildung oder eine $\mathrm{Zu}$ satzbezeichnung kann einen qualifikationsbezogenen Sonderbedarf begründen, wenn sie den in $\$ 37$ Abs. 1 BedarfsplanungsRL genannten Qualifikationen vom zeitlichen und qualitativen Umfang her gleichsteht.

4. Bei der Bedarfsprüfung sind nur reale, nicht dagegen potenzielle Versorgungsangebote zu berücksichtigen, die tatsächlich nicht zur Verfügung stehen, weil Leistungserbringer nicht zur Erbringung weiterer Leistungen bereit oder tatsächlich nicht in der Lage sind.

5. Bei der Feststellung von Sonderbedarf haben die Zulassungsgremien gemä $\$ 36$ Abs. 3 Nr. 1 BedarfsplanungsRL zur Feststellung einer unzureichenden Versorgungslage zunächst eine Region abzugrenzen, die vom beantragten Ort der Niederlassung aus versorgt werden soll und die dortige Versorgungslage zu bewerten.

6. Der Versorgungsbedarf am beantragten Ort allein begründet noch keinen qualifikationsbezogenen Sonderbedarf. Entscheidend ist, ob dieser nicht durch andere (zumutbar erreichbare) Praxen gedeckt werden kann, auch wenn dies bedeutet, dass die Versicherten nicht an ihrem Wohnort oder in unmittelbarer Nähe ihres Wohnortes behandelt werden.

7. Ohne Bedeutung ist, ob sich die Patienten die Behandlung gerade an einem bestimmten Ort oder beson-

\section{Eingesandt und bearbeitet}

von Rechtsanwalt und Notar Dr. iur. Paul Harneit,

Fachanwalt für Medizinrecht, CausaConcilio Rechtsanwälte, Deliusstraße 16, 24114 Kiel, Deutschland 